\title{
Las aulas virtuales en el proceso de enseñanza y aprendizaje de la Estadística
}

\author{
Virtual classrooms in the teaching and learning process of Statistics \\ Salas de aula virtuais no processo de ensino e aprendizagem de Estatísticas \\ Dayana Alejandra Barrera ${ }^{1}$ \\ Nidia Danigza Lugo-López²
}

Recibido: enero de 2019

Aceptado: abril de 2019

Para citar este artículo: Barrera, J.A.; Lugo-López, N.D. (2019). Las aulas virtuales en el proceso de enseñanza y aprendizaje de la Estadística. Revista Científica, 35(2), 183-191. Doi: https://doi.org/10.14483/23448350.14368

\section{Resumen}

En la actualidad se innova en la investigación acerca de los métodos de enseñanza en estadística y probabilidad. Sin embargo, es necesario investigar el proceso de aprendizaje de esta asignatura dentro de entornos virtuales. Por esta razón, se pretende observar cómo han cambiado los métodos de enseñanza de la asignatura a través del tiempo en la Universidad Manuela Bertrán (UMB) Virtual, tomando las notas de las evaluaciones finales de dos grupos de estudiantes de estadísticas de las aulas virtuales. Utilizando la prueba T se realizó un análisis comparativo de las aulas, concluyendo que el método de enseñanza en el que se usaron diferentes recursos didácticos muestra un aprendizaje superior.

Palabras clave: aprendizaje, enseñanza, estadística, evaluación continua, foro académico, prueba T, recursos didácticos.

\begin{abstract}
Currently, research is being innovated on teaching methods in statistics and probability, however, it is necessary to investigate the learning process on this subject in virtual environments. For this reason, it is intended to observe how the teaching methods of the subject have changed over time at the Manuela Beltrán Virtual University (UMB) Virtual, taking the notes of the final evaluations of two groups of students of statistics of the virtual classrooms. Using the $\mathrm{T}$ test, a comparative analysis of the classrooms is carried out, concluding that the teaching method in which different.
\end{abstract}

Keywords: academic forum, continuous evaluation, didactic resources, learning, statistics, T-test, teaching. 


\section{Resumo}

Atualmente pesquisas estão a ser inovadas em métodos de ensino em estatística e probabilidade, no entanto, é necessário investigar o processo de aprendizagem deste assunto em ambientes virtuais. Por esta razão, pretende-se observar como os métodos de ensino do tema mudaram ao longo do tempo na Universidade Virtual Manuela Bertrán (UMB) Virtual, tomando as notas das avaliações finais de dois grupos de estudantes de estatística das salas de aula virtuais. Utilizando o teste T, é realizada uma análise comparativa das salas de aula, concluindo que o método de ensino em que diferentes recursos didáticos foram utilizados mostra uma aprendizagem superior.

Palavras-chaves: recursos didáticos, fórum acadêmico, avaliação contínua, T-test, ensino, aprendizagem, estatística.

\section{Introducción}

Al enseñar estadística se espera que los estudiantes sean capaces de emplear los conocimientos adquiridos en sus campos laborales y que estos nuevos saberes les permitan solucionar problemas de su vida diaria o profesional. Para lograr este objetivo en el aprendizaje de conceptos de la estadística, es necesario que la metodología de enseñanza se centre en la interpretación y aplicación de situaciones prácticas (Gómez y Rivera, 2015). También es importante que el alumno sea un ente activo en su proceso de enseñanza-aprendizaje y que el aprendizaje no que se reduzca a una visión transmisionista.

Ahora bien, ¿cómo lograr esto desde la virtualidad? Debido a que actualmente la Universidad Manuela Beltrán (UMB) imparte programas académicos virtuales, en la sede ubicada en Cajicá-Cundinamarca, que cuenta con un Departamento de Ciencias Básicas el cual orienta todas las asignaturas del campo de las matemáticas y ciencias naturales, entre ellas la estadística y probabilidad. Para esto, es necesario el uso de una plataforma para la administración del aprendizaje, comúnmente denominada aula virtual.
Antes de continuar, es importante aclarar qué se entiende en esta investigación por aula virtual. Esta se define como:

Una herramienta que brinda las posibilidades de realizar enseñanza en línea. Es un entorno privado que permite administrar procesos educativos basados en un sistema de comunicación mediado por computadoras. De manera que se entiende como Aula Virtual, al espacio simbólico en el que se produce la relación entre los participantes en un proceso de enseñanza y aprendizaje que, para interactuar entre sí y acceder a la información relevante, utilizan prioritariamente un sistema de comunicación mediada por computadoras. (UCAB, 2013, p. 1)

Para la adecuada realización de la enseñanza desde el aula virtual, esta cuenta con diferentes herramientas entre la cuales se encuentran: información general, documentación del curso, tareas o asignaciones, chat, correo electrónico, foros, ejercicios interactivos, cuestionarios, entre otros. Algunas de estas herramientas tienen por objetivo facilitar la comunicación entre el docente y el estudiante; estas a su vez se pueden dividir en comunicación sincrónica (chat) y asincrónica (foros, correo electrónico).

En este trabajo se pretende, por lo tanto, observar cómo el uso o no de los diferentes recursos descritos en el párrafo anterior en las aulas virtuales de la asignatura de estadística y probabilidad, provoca mejores resultados en el desempeño académico de los estudiantes. Para lograr estudiar el impacto del uso de las herramientas virtuales se comparan los resultados académicos de dos aulas virtuales ya terminadas por los estudiantes.

Estas fueron escogidas ya que desde la creación de las aulas virtuales de estadística y probabilidad en el 2010 hasta el año 2015, todas estas utilizaban la misma metodología. Esta trataba de dividir el aula en tres cohortes o temáticas, en los que se colocaban tres talleres de ejercicios 
respectivamente y las notas de estos se promediaron para obtener las notas finales de los estudiantes, dejando de lado el uso de herramientas como chat y foros. En cambio, a partir de la primera aula del 2016, se realizó una renovación curricular de la asignatura que reestructuró las actividades evaluativas, pues, aparte de los talleres de ejercicios se propuso los foros académicos de discusión de determinadas temáticas y la evaluación en línea con preguntas múltiples, realizada en un tiempo determinado. Por lo que esta investigación le permitió a las investigadoras determinar el impacto del uso de foro en las aulas virtuales, se dejaron de lado las otras herramientas ya que no fueron usadas en el aula virtual.

\section{Importancia de la comunicación asincró- nica en la enseñanza: foro}

Resultado de la sociedad de la información en la que actualmente se encuentra inmersa la población mundial, se han desarrollado diferentes herramientas de comunicación mediadas por el internet, como lo afirman Ramírez y Rama:

las sociedades de la información han promovido nuevas formas de comunicación mediante herramientas de comunicación sincrónica (como chat, videoconferencias, entre otros) y asincrónica (foros, correo electrónico, entre otros), dando origen a nuevos conceptos cómo Ambientes Virtuales de Aprendizaje (AVA), aprendizaje colaborativo, cooperativo, e-learning, entre otros. (2014, p. 36)

Autores como Buil, Hernández, Sesé y Urquizu (2011) señalan que el foro le permite al estudiante publicar un mensaje en cualquier momento, quedando siempre visible, para que posteriormente cualquier otro participante pueda consultarlo y contestar si lo estima oportuno. Esto permite mayor tiempo de reflexión para dar respuesta a las situaciones problema propuestas.
Así mismo, el foro permite generar discusiones alrededor de una temática, presentar los puntos de vista de los participantes libremente para lograr un trabajo colaborativo y una retroalimentación constante, que puede o no venir del docente de la asignatura, reforzando también el aprendizaje desde la enseñanza o los otros (Cerrudo y Ferreyra, 2015).

Todo lo anterior convierte al foro en una importante herramienta de comunicación asincrónica, dándole libertad al estudiante para escoger el momento, el ritmo y el lugar de estudio, independiente del docente y de los compañeros (Chaves Torres, 2017).

\section{Prueba T de Student}

Para el análisis de los resultados de esta investigación se hizo uso de la prueba $\mathrm{T}$, por lo que se hace necesario explicar en qué consiste. Esta es una prueba estadística que toma dos grupos con variables cuantitativas (en este caso las notas de los estudiantes) y compara las medias de cada grupo, determinando si son significativamente iguales (hipótesis nula) o significativamente distintas (hipótesis alternativa). Esta tiene una confianza del $95 \%$ de que la decisión que se tome realmente sea correcta, y un nivel de significancia del $5 \%$ de que sea errónea. El uso de la prueba $\mathrm{T}$ permite determinar si se rechaza o no la hipótesis nula.

\section{Metodología}

El presente estudio busca determinar el impacto que tiene el uso de los foros (herramienta de comunicación asincrónica) y las evaluaciones continuas en la compresión de los contenidos de la asignatura de estadística y probabilidad en el aula virtual. Con el propósito de lograr esto se utilizó un análisis cuantitativo tomando como herramienta la prueba T para muestras independientes, para comparar los resultados en sus notas de dos grupos de estudiantes que vieron la asignatura. A 
continuación, se explican las actividades realizadas al interior de las aulas virtuales y lo referente al grupo de estudiantes 1 y al grupo de estudiantes 2 .

\section{Actividades realizadas en las aulas virtuales}

Como se indicó en párrafos anteriores, esta investigación cuenta con dos grupos de estudiantes, los cuales se identifican como grupo 1 y grupo 2. En los dos grupos se tenía por objetivo cumplir con el diseño curricular de la asignatura que está dividido en dos temáticas principales: estadística descriptiva (recolección de información, representación gráfica, medidas descriptivas y análisis de la información) y probabilidad básica (definición de las medidas de probabilidad, propiedades de las probabilidades, teoría del conteo, probabilidades con condicionales y regla de Bayes).

Las características personales y académicas de los grupos eran equivalentes, ya que se tenían personas con edades entre 30 y 50 años, pertenecientes a los estratos 2 y 3; además, el $70 \%$ de la muestra en los dos grupos vive fuera de Bogotá y se encontraban laborando. Las actividades realizadas en las aulas se describen en las tablas 1 y 2 .

Tabla 1. Actividades realizadas en el aula con el grupo 1.

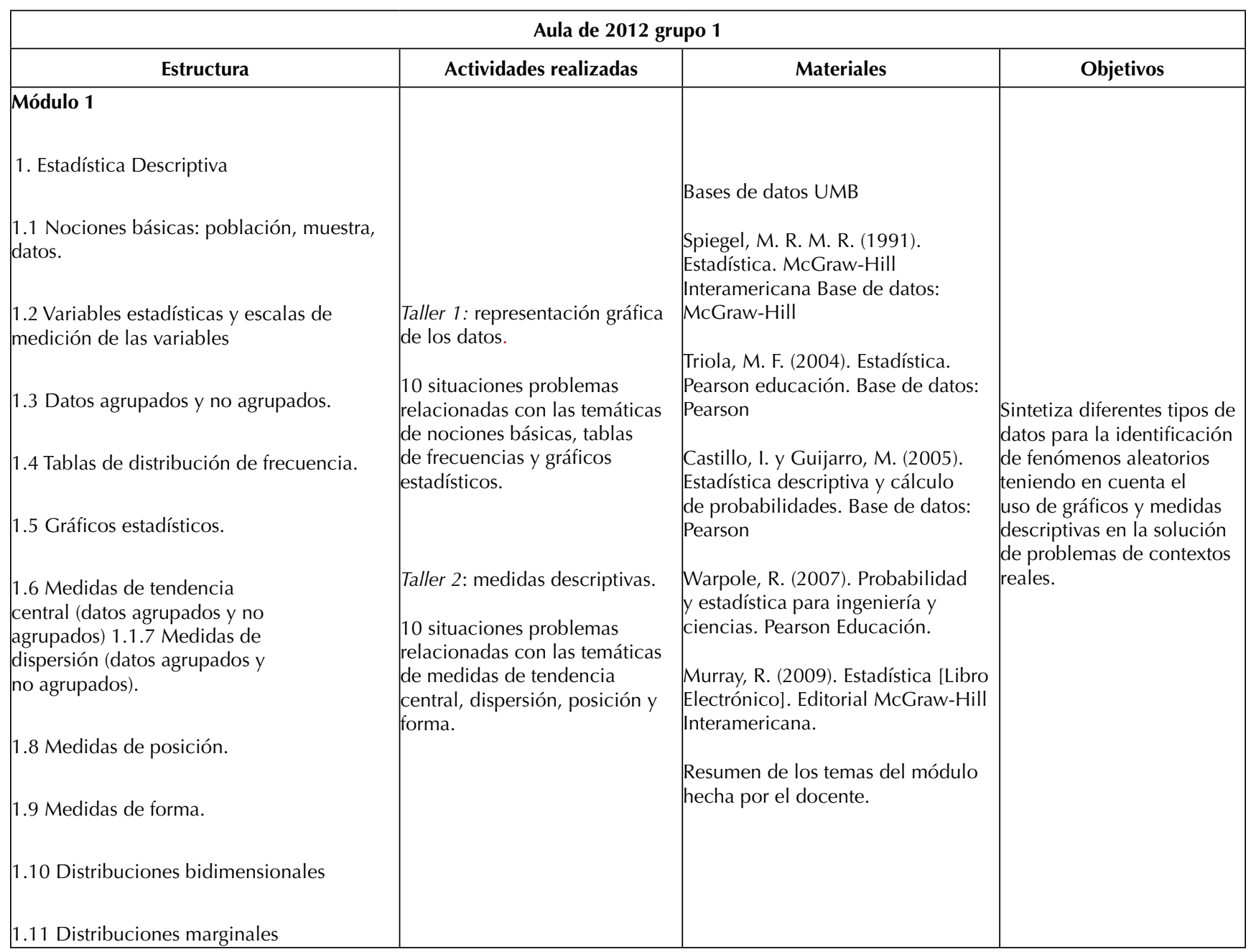




\begin{tabular}{|c|c|c|c|}
\hline $\begin{array}{l}\text { Módulo } 2 \\
\text { 2. Probabilidad y combinatoria } \\
\text { 2.1 } \begin{array}{l}\text { Nociones básicas: experimentos } \\
\text { aleatorios, espacio muestral, } \\
\text { sucesos. }\end{array} \\
\text { 2.2 Operaciones con sucesos, sucesos } \\
\text { compatibles e incompatibles. } \\
\text { 2.3 Combinatoria: permutaciones, } \\
\text { combinaciones y variaciones } \\
\text { 2.4 Definición de probabilidad simple. } \\
\text { 2.5 Probabilidad condicional } \\
\text { 2.6 Teoremas de probabilidad total y } \\
\text { Bayes }\end{array}$ & $\begin{array}{l}\text { Taller 3: probabilidad básica } \\
10 \text { situaciones problemas } \\
\text { relacionadas con todas las } \\
\text { temáticas de este módulo. }\end{array}$ & $\begin{array}{l}\text { Bases de datos UMB } \\
\text { Spiegel, M. R. M. R. (1991). Estadística. } \\
\text { McGraw-Hill Interamericana Base de } \\
\text { datos: McGraw-Hill } \\
\text { Triola, M. F. (2004). Estadística. Pearson } \\
\text { educación. Base de datos: Pearson } \\
\text { Castillo, I. y Guijarro, M. (2005). Es- } \\
\text { tadística descriptiva y cálculo de proba- } \\
\text { bilidades. Base de datos: Pearson } \\
\text { Warpole, R. (2007). Probabilidad y } \\
\text { estadística para ingeniería y ciencias. } \\
\text { Pearson Educación. } \\
\text { Murray, R. (2009). Estadística [Libro } \\
\text { Electrónico]. Editorial McGraw-Hill } \\
\text { Interamericana. } \\
\text { Resumen de los temas del módulo hecha } \\
\text { por el docente. } \\
\text { Los materiales disponibles en los dos } \\
\text { módulos. }\end{array}$ & $\begin{array}{l}\text { Aplica los conceptos de la } \\
\text { teoría de las probabilidades para } \\
\text { abordar fenómenos aleatorios, } \\
\text { mediante el uso de la regla } \\
\text { de Bayes en la solución de } \\
\text { situaciones problema. }\end{array}$ \\
\hline Evaluación final & \begin{tabular}{|l|} 
La evaluación era de 10 \\
preguntas de opción múltiple \\
con contextualizadas alineadas \\
a cada uno de los temas, dados \\
en los módulos 1 y 2 .
\end{tabular} & & $\begin{array}{l}\text { Conocer el desempeño de los } \\
\text { estudiantes, al enfrentarse a } \\
\text { situaciones problema que están } \\
\text { relacionadas con las temáticas } \\
\text { de la asignatura. }\end{array}$ \\
\hline
\end{tabular}

Fuente: Elaboración propia de los autores.

Tabla 2. Actividades realizadas en el aula con el grupo 2 .

\begin{tabular}{|c|c|c|c|}
\hline \multicolumn{4}{|c|}{ Aula de 2016 grupo 2} \\
\hline Estructura & Actividades realizadas & Materiales & Objetivos \\
\hline $\begin{array}{l}\text { Módulo } 1 \\
\text { 1. Estadística descriptiva } \\
\text { 1.1 Nociones básicas: población, } \\
\text { muestra, datos. } \\
\text { 1.2 Variables estadísticas y escalas de } \\
\text { medición de las variables } \\
\text { 1.3 Datos agrupados y no agrupados. } \\
\text { 1.4 Tablas de distribución de frecuencia. } \\
\text { 1.5 Gráficos estadísticos. } \\
\text { 1.6 Medidas de tendencia } \\
\text { central (datos agrupados y no } \\
\text { agrupados) } 1.1 .7 \text { Medidas de } \\
\text { dispersión (datos agrupados y } \\
\text { no agrupados) } \\
\text { 1.8 Medidas de posición. } \\
\text { 1.9 Medidas de forma. } \\
\text { 1.10 Distribuciones bidimensionales } \\
\text { 1.11 Distribuciones marginales }\end{array}$ & $\begin{array}{l}\text { Actividad práctica: } \\
\text { Laboratorio } 1 \text { encuesta de } \\
\text { investigación usando } \\
\text { herramientas de estadística } \\
\text { descriptiva con Excel } \\
\text { Actividad teórica 1: } \\
\text { Evaluación módulo } 1 \\
\text { La evaluación cuenta } \\
\text { con } 10 \text { preguntas de } \\
\text { opción múltiple con } \\
\text { contextualizadas alineadas } \\
\text { a cada uno de los subtemas } \\
\text { tratados en el módulo }\end{array}$ & $\begin{array}{l}\text { Bases de datos UMB } \\
\text { Spiegel, M. R. M. R. (1991). } \\
\text { Estadística. McGraw-Hill } \\
\text { Interamericana Base de datos: } \\
\text { McGraw-Hill } \\
\text { Triola, M. F. (2004). Estadística. } \\
\text { Pearson educación. Base de } \\
\text { datos: Pearson } \\
\text { Castillo, I. y Guijarro, M. (2005). } \\
\text { Estadística descriptiva y cálculo } \\
\text { de probabilidades. Base de da- } \\
\text { tos: Pearson } \\
\text { Warpole, R. (2007). Probabilidad } \\
\text { y estadística para ingeniería y } \\
\text { ciencias. Pearson Educación. } \\
\text { Murray, R. (2009). Estadística } \\
\text { [Libro Electrónico]. Editorial } \\
\text { McGraw-Hill Interamericana. } \\
\text { Resumen de los temas del } \\
\text { módulo hecha por el docente. } \\
\text { Video Clase: es una sesión de } \\
\text { video clase sincrónica, que } \\
\text { programa el docente, en el cual } \\
\text { se esperan ampliar algunas } \\
\text { temáticas de los módulos y se } \\
\text { solucionen inquietudes precisas } \\
\text { manifestadas por los estudiantes } \\
\text { participantes en la sesión. }\end{array}$ & $\begin{array}{l}\text { Sintetiza diferentes tipos de } \\
\text { datos para la identificación } \\
\text { de fenómenos aleatorios } \\
\text { teniendo en cuenta el } \\
\text { uso de gráficos y medidas } \\
\text { descriptivas en la solución } \\
\text { de problemas de contextos } \\
\text { reales. }\end{array}$ \\
\hline
\end{tabular}




\begin{tabular}{|c|c|c|c|}
\hline $\begin{array}{l}\text { Módulo } 2 \\
\text { 2. Probabilidad y } \\
\text { combinatoria } \\
\text { 2.1 Nociones } \\
\text { básicas: experimentos } \\
\text { aleatorios, espacio } \\
\text { muestral, sucesos. } \\
\text { 2.2 Operaciones } \\
\text { con sucesos, sucesos } \\
\text { compatibles e } \\
\text { incompatibles. } \\
\text { 2.3 Combinatoria: } \\
\text { Permutaciones, } \\
\text { combinaciones y } \\
\text { variaciones } \\
\text { 2.4 Definición de } \\
\text { probabilidad simple. } \\
\text { 2.5 Probabilidad } \\
\text { condicional } \\
\text { 2.6 Teoremas de } \\
\text { probabilidad total y } \\
\text { Bayes }\end{array}$ & $\begin{array}{l}\text { Actividad teórica 2: Foro Probabilidad y } \\
\text { Combinatoria. } \\
\text { 1. Participar en el foro significa realizar } \\
\text { tres ejercicios de la guía asociadas a } \\
\text { temas de probabilidad y combinatorias. } \\
\text { Estos se socializarán a partir de una } \\
\text { imagen con procesos y usando de } \\
\text { editor ecuaciones. } \\
\text { 2. Retroalimentar el proceso de los } \\
\text { compañeros mediante ejemplos de las } \\
\text { bases de datos o correcciones de los } \\
\text { ejercicios. } \\
\text { 3. Realiza las correcciones sugeridas } \\
\text { por el docente o compañeros de los } \\
\text { ejercicios si son necesarias. } \\
\text { El docente realiza la revisión de } \\
\text { cada una de las participaciones y } \\
\text { retroalimenta el proceso académico. } \\
\text { Actividad teórica 3: Evaluación módulo } \\
2 \\
\text { La evaluación cuenta con } 10 \\
\text { preguntas de opción múltiple con } \\
\text { contextualizadas alineadas a cada uno } \\
\text { de los subtemas tratados en el módulo. }\end{array}$ & $\begin{array}{l}\text { Bases de datos UMB } \\
\text { Spiegel, M. R. M. R. (1991). Estadística. } \\
\text { McGraw-Hill Interamericana Base de } \\
\text { datos: McGraw-Hill } \\
\text { Triola, M. F. (2004). Estadística. Pear- } \\
\text { son educación. Base de datos: Pearson } \\
\text { Castillo, I. y Guijarro, M. (2005). } \\
\text { Estadística descriptiva y cálculo de } \\
\text { probabilidades. Base de datos: Pearson } \\
\text { Warpole, R. (2007). Probabilidad y } \\
\text { estadística para ingeniería y ciencias. } \\
\text { Pearson Educación. } \\
\text { Murray, R. (2009). Estadística [Libro } \\
\text { Electrónico]. Editorial McGraw-Hill } \\
\text { Interamericana. } \\
\text { Resumen de los temas del módulo } \\
\text { hecha por el docente. } \\
\text { Video Clase: es una sesión de video } \\
\text { clase sincrónica, que programa el } \\
\text { docente, en el cual se esperan ampliar } \\
\text { algunas temáticas de los módulos y } \\
\text { se solucionen inquietudes precisas } \\
\text { manifestadas por loe estudiantes par- } \\
\text { ticipantes en la sesión. }\end{array}$ & $\begin{array}{l}\text { Aplica los conceptos de la } \\
\text { teoría de las probabilidades } \\
\text { para abordar fenómenos } \\
\text { aleatorios, mediante el uso } \\
\text { de la regla de Bayes en la } \\
\text { solución de situaciones } \\
\text { problema. }\end{array}$ \\
\hline Evaluación final & $\begin{array}{l}\text { La evaluación era de } 10 \text { preguntas de } \\
\text { opción múltiple con contextualizadas } \\
\text { alineadas a cada uno de los temas, } \\
\text { dados en los módulos } 1 \text { y } 2 \text {. }\end{array}$ & $\begin{array}{l}\text { Los materiales disponibles en los dos } \\
\text { módulos. }\end{array}$ & $\begin{array}{l}\text { Conocer el desempeño de } \\
\text { los estudiantes, al enfrentarse } \\
\text { a situaciones problema que } \\
\text { están relacionadas con las } \\
\text { temáticas de la asignatura. }\end{array}$ \\
\hline
\end{tabular}

Fuente: Elaboración propia de los autores.

\section{Participantes e instrumentos de recolección de información}

Las actividades se pusieron en marcha durante dos periodos de tiempo no consecutivos 2012 y 2016, con estudiantes de ingeniería de software modalidad virtual, pertenecientes a la Universidad Manuela Beltrán que hacen parte del campus virtual de la misma. En total, se contó con 19 estudiantes para el 2012 (grupo 1) y 46 estudiantes en el 2016 (grupo 2). La diferencia principal entre estos grupos radica en que en el aula de 2012 solamente se realizaron talleres para la evaluación final de los estudiantes, lo que se siguió haciendo hasta el aula del 2016, donde por primera vez se agregaron como actividades evaluativas los foros académicos.

Los foros funcionaban de la siguiente manera: se colocó un taller con preguntas y problemas relacionados a los temas del módulo dando instrucciones al estudiante sobre dónde debían realizar dos problemas y dos retroalimentaciones a sus compañeros, sin repetir soluciones o retroalimentaciones. El tutor calificaba las soluciones y era el moderador en las retroalimentaciones, estas pueden ser soluciones alternativas, aportes nuevos al problema o correcciones a las soluciones propuestas.

Un ejemplo del tipo de preguntas que se utilizaron en los talleres o foros son:

La probabilidad de que un hombre viva 20 años es $1 / 4$ y la de que su mujer viva 20 años es $1 / 3$. Se pide calcular la probabilidad de que ambos vivan 20 años. Interprete adecuadamente su respuesta.

Como se observa, no solamente se pidió el cálculo, sino que se hiciera la interpretación del resultado; en este caso la repuesta es $1 / 4 * 1 / 3=1 / 12$, con lo que se interpretó como que la probabilidad 
de que hombres y mujeres vivan 20 años es de 1/12, o en porcentaje del $8.3 \%$.

El objetivo de estos talleres era fortalecer en el estudiante la capacidad de resolver problemas, haciendo un análisis adecuado de la situación que le permitiera discriminar entre las fórmulas la correcta, realizar los cálculos correctos y, además, interpretar esta respuesta en un entorno real. Ambos conceptos se tenían en cuenta a la hora de evaluar las preguntas; así, el foro fue para los estudiantes un espacio para discutir la solución a los problemas planteados y recibir retroalimentación, el cual no tenían antes del uso del foro.

\section{Resultados}

Con el propósito de realizar una comparación entre los resultados académicos de los dos grupos y poder medir el impacto del foro en las actividades realizadas, se usó la prueba T. Esta se aplicó a las notas finales obtenidas por los estudiantes, la cual es resultado del promedio de las notas parciales. Se debe recordar que lo único que cambió en las dos aulas virtuales fue el uso del foro.

Antes de continuar con los detalles de la prueba T, se debe explicar qué procesos de depuración se realizaron en los datos y cómo se hizo el estudio de normalidad de los mismos. Se tomaron los datos de los estudiantes que participaron en todas las actividades así:

1. El grupo I contaba con 19 estudiantes

2. En el grupo I fueron eliminados 4 estudiantes $(21 \%)$

3. El grupo I quedó con 15 estudiantes (79 \%)

4. El grupo II tenía 46 estudiantes

5. En el grupo II fueron eliminados 13 estudiantes $(28 \%)$

6. El grupo II quedó con 33 estudiantes (72\%)

La prueba $\mathrm{T}$ permite comparar los promedios entre dos muestras pareadas o independientes, teniendo igualdad o no en las varianzas. Como es una prueba paramétrica, solamente se puede aplicar cuando los datos se distribuyen normalmente.

Según Gómez y Soria, "Hay que tener siempre en cuenta que las pruebas paramétricas son más potentes y dan más información que los no paramétricos, por lo que, sí pueden usarse, se prefieren" (2001, p. 1). Por esta razón, se utilizaron las pruebas Shapiro-Wilks (para la muestra del grupo I que tiene menos de 30 estudiantes) y Kolmogorov-Smirnov (para la muestra del grupo II que tiene más de 30 estudiantes), para comprobar la normalidad de los datos.

Pruebas de normalidad:

$$
\begin{gathered}
H_{0}: \text { Lamuestraprovienedeunapoblacióndistribuidanormalmente } \\
H_{a}: \text { Lamuestranoprovienedeunapoblacióndistribuidanormalmente }
\end{gathered}
$$

Tabla 3. Pruebas de normalidad grupos I y II.

\begin{tabular}{llll}
\hline & P-valor $K S^{1}$ & P-valor $S W^{2}$ & \\
\cline { 2 - 3 } Grupo I & 0.078 & $a^{3}$ \\
Grupo II & 0.2 & 0.287 & b \\
\hline
\end{tabular}

(1) KS: Kolmogorov-Smirnov, (2) SW: Shapiro-Wilk. (3) Letras diferenciadas indican diferencias significativas $\alpha=0.05$

Fuente: Elaboración propia de los autores. 
En la tabla 3 se presentan los resultados de los test a los dos grupos. Tomando como nivel de significancia del 0.05, como los p-valores en las pruebas Shapiro-Wilk y Kolmogorov-Smirnov son mayores a 0.05 , no se rechaza $H_{0}$.

Se obtuvo que ambas muestras provienen de poblaciones que se distribuyen normalmente. Con los resultados anteriores, se tiene la normalidad en los datos, permitiendo aplicar la prueba T. Las hipótesis nula y alternativa son:

$H_{0}$ : los estudiantes sometidos al método de enseñanza virtual del 2016 obtuvieron los mismos resultados que los estudiantes sometidos al método de enseñanza virtual del 2012. $\left(\mu_{I}=\mu_{I l}\right)$.

$H_{a}$ : los estudiantes sometidos al método de enseñanza virtual del 2016 obtuvieron mejores resultados que los estudiantes sometidos al método de enseñanza virtual del 2012. $\left(\mu_{I}<\mu_{I l}\right)$.

Los resultados de la prueba se muestran en la tabla 4, en la que se puede ver que los límites de confianza son negativos asumiendo o no varianzas iguales, es decir:

$$
\mu_{I}-\mu_{I I}<0 \text { entonces } \mu_{I}<\mu_{I I}
$$

Por lo tanto, se rechaza $H_{0}$, debido a que cuando suponemos varianzas iguales o distintas obtenemos que los límites de confianza son negativos, como lo señala la tabla, con lo que existen evidencias estadísticas que permiten afirmar con un $5 \%$ de significación que "los estudiantes sometidos al método de enseñanza virtual del 2016 obtuvieron mejores resultados que los estudiantes sometidos al método de enseñanza virtual del 2012".

\section{Discusión}

En la literatura se pueden encontrar experiencias previas con respecto al foro académico virtual, considerándolo una herramienta pedagógica enriquecedora, para el conocimiento que impulsa la participación de los estudiantes, permitiendo que intercambien ideas, establezcan posturas que se descartan o se asumen parcialmente, dando aportes con nuevas ideas, ejemplos, sitios de internet, bibliografía, entre otros (Buil, Hernández, Sesé y Urquizu, 2011). Esto es importante cuando la enseñanza se hace desde la virtualidad.

Uno de los recursos didácticos que ha generado óptimos resultados en el aprendizaje de los contenidos de la asignatura es el foro académico. Y una de las ventajas que posee es la comunicación asincrónica, teniendo en cuenta que a los estudiantes de la modalidad virtual les es difícil estar de manera sincrónica en la participación de las actividades, por sus trabajos y responsabilidades; con lo que este aspecto es una gran ventaja a la hora de participar en una actividad académica.

En el foro se genera un debate que produce de manera natural herramientas de "enriquecimiento académico", que permiten retroalimentar el proceso de aprendizaje de los contenidos en cualquier asignatura del conocimiento, haciendo que en el área de estadística se genere la polémica y el interés por la participación e interacción de los temas propuestos. De esta manera, puede afirmarse que parte de los buenos resultados en el grupo II se debe al foro académico del módulo I, afirmación que es confirmada con los resultados de la prueba $\mathrm{T}$.

Tabla 4. Pruebas T diferencia de medias de los grupos I y II.

\begin{tabular}{|c|c|c|c|c|}
\hline & $P V^{1}$ & $L I C^{2}$ & $L S C^{3}$ & \\
\hline Suponiendo iguales varianzas & 0.016 & -1.0439 & -0.112 & $a^{4}$ \\
\hline Suponiendo distintas varianzas & 0.027 & -1.0845 & -0.070 & $\mathrm{~b}$ \\
\hline
\end{tabular}

Fuente: Elaboración propia de los autores. 
Desde la experiencia en las aulas de estadística de la UMB Virtual, el foro ha permitido abordar los temas de manera natural, haciendo que los estudiantes practiquen ejercicios, retroalimenten el proceso de sus compañeros y puedan corregir y aclarar dudas con la ayuda del tutor. Esta experiencia se explica por la naturaleza asincrónica de un foro académico, dando lugar a que se tome más tiempo para la participación, la retroalimentación y la reflexión del aprendizaje de los contenidos

Así, los resultados obtenidos del grupo II con respecto al grupo I en parte se deben a que en el grupo I solamente realizó algunos talleres y una evaluación final; a diferencia del grupo II en el que se realizaron varios foros, talleres y evaluación final, haciendo un seguimiento más profundo acerca del progreso del aprendizaje de los estudiantes, durante el transcurso del curso. Resultados similares son encontrados por Cerrudo y Ferreyra, L. (2015), lo que reafirma las conclusiones encontradas en este trabajo, en el aula virtual es necesario usar herramientas de comunicación asincrónica que le permite al estudiante compartir su conocimiento con sus compañeros y con el docente.

\section{Conclusiones}

En esta investigación pudimos concluir que herramientas como el foro académico o la evaluación en línea obtuvieron mejores resultados en el promedio de notas de los estudiantes.

Es importante desarrollar recursos académicos que permitan mejorar la interactividad entre los participantes de las aulas virtuales de estadística y probabilidad, en los que se intensifique los trabajos colaborativos, la retroalimentación constante del tutor y el seguimiento al progreso académico de los estudiantes.

El foro, al ser una herramienta que permite proponer diferentes soluciones a un problema, en este caso estadístico, propicia la discusión alrededor de la temática, lo que genera un pensamiento crítico en cada uno de los participantes.

\section{Referencias}

Buil, I.; Hernández, B; Sesé J.; Urquizu, P. (2011). Los foros de discusión y sus beneficios en la docencia virtual: recomendaciones para un uso eficiente. Revista Innovar, 22(43), 131-144.

Cerrudo, A.; Ferreyra, L. (2015). Importancia del uso de foros como herramienta de comunicación, formación y potenciación del aprendizaje en aulas virtuales en la educación superior a distancia. En III Jornadas de TIC e Innovación en el Aula. http://sedici.unlp.edu.ar/ handle/10915/48785

Chaves-Torres, A. (2017). La educación a distancia como respuesta a las necesidades educativas del siglo XXI. Revista Academia y Virtualidad, 10(1), 23-41. https://doi. org/10.18359/ravi.2241

Gómez-Biedma, S.; Vivó, M.; Soria, E. (2001). Pruebas de significación en Bioestadística. Revista de Diagnóstico Biológico, 50(4), 1.

Moya, M. (2008). La utilización de los foros en la enseñanza de la matemática mediada por tecnología digital (tesis maestría). Universidad Nacional de La Plata. Argentina. https://goo.gl/ Ar9TTC

Ramírez, F; Rama, C. (2014). Los recursos de aprendizaje en educación a distancia: nuevos escenario, experiencias y tendencias. Lima: Fondo Editorial.

UCAB (2013). ¿Qué es un aula virtual?. Curso continental AUSJAL sobre pobreza en América Latina. Universidad Católica Andrés Bello. Caracas, Venezuela. http://apps.ucab.edu.ve/diplomadonew/aulavirtual.pdf

Vides, S; Rivera, J. (2015). La ingeniería didáctica en el proceso de enseñanza y aprendizaje de la estadística. Omnia, 21(2), 96-104. 